\title{
ACÚMULO DE CARGAS ELÉTRICAS EM PROPELENTE SÓLIDO COMPÓSITO COM MATRIZ DE POLIBUTADIENO LÍQUIDO HIDROXILADO
}

\author{
Silvio Manea*, Gabriela Knippelberg B. Manea, Koshun Iha e José A. F. F. Rocco \\ Departamento de Química, Instituto Tecnológico da Aeronáutica, Praça Marechal Eduardo Gomes, 50, Vila das Acácias, \\ 12228-900 São José dos Campos - SP, Brasil
}

Recebido em 28/02/2013; aceito em 08/08/2013; publicado na web em 24/09/2013

\begin{abstract}
ACCUMULATION OF ELECTRIC CHARGES IN HYDROXYL-TERMINATED POLYBUTADIENE BASED COMPOSITE SOLID PROPELLANT. Composite solid propellants prepared with HTPB prepolymer - Hydroxyl Terminated Polybutadiene, AP - Ammonium Perchlorate as oxidizer and aluminum particles as an additive metal, have characteristics of high electrical resistivity. The loading process of the polymer matrix did not obtain homogeneity, resulting in clusters, mainly of metal particles. The effect of clustering in the composite was studied and observed experimentally, and this effect was one of the factors explaining the phenomenon of electrical charging of the composite. This electrical potential, when discharged abruptly, can generate an electric spark with sufficient energy for sustained ignition of a solid rocket motor.
\end{abstract}

Keywords: composite solid propellant; static-charge accumulation; electrostatic discharge.

\section{INTRODUÇÃO}

A propulsão química utilizada em lançadores de satélites, espaçonaves e mísseis é designada como motores aeroespaciais que utilizam a energia das ligações químicas produzida inicialmente pela decomposição térmica das espécies químicas dos propelentes sólidos, líquidos ou híbridos. A configuração do propelente e do oxidante é diversa em cada motor e aplicável de acordo com as necessidades e características da missão a ser desenvolvida pelo engenho. $\mathrm{O}$ motor híbrido, normalmente, é uma configuração composta por um combustível sólido instalado numa câmara de combustão na qual é injetado o oxidante que pode estar no estado líquido ou gasoso. A reação de combustão, por ser exotérmica, produz gases quentes que ao expandirem são direcionados para a saída do tubo motor foguete onde está localizado o convergente-divergente do motor, produzindo assim empuxo. Na concepção do motor foguete com propulsão líquida, os propelentes, combustível e oxidante são armazenados separadamente no estado líquido, sendo bombeados para a câmara de combustão do motor onde ocorre a queima. Da mesma forma, essa queima gera gases quentes, e consequentemente, empuxo.

Os motores foguetes que utilizam propelentes sólidos do tipo compósito são empregados como base para sistemas de propulsão otimizados, onde o volume de energia acumulado permite usar um engenho de menor porte quando comparado ao híbrido ou líquido. Nesse caso, o controle da velocidade da queima é exercido por meio da medida linear de queima do propelente, a qual é dependente da composição físico-química dos ingredientes do propelente, da geometria do grão e da pressão na câmara de combustão durante a operação do motor. ${ }^{1}$

Os propelentes sólidos são composições onde matrizes poliméricas são carregadas sob a forma de um material compósito com uma quantidade elevada de partículas sólidas dispersas de forma aleatória nessa mesma matriz. No processamento do material, busca-se distribuir essas partículas sólidas, constituídas principalmente por espécies combustíveis e oxidantes, de forma homogênea para que ocorra um processo de combustão regular pela intima proximidade entre as espécies químicas envolvidas. Para obter uma força propulsiva

*e-mail: silvio.manea@gmail.com maximizada utiliza-se uma quantidade mínima do polímero, suficiente para preencher os vazios entre as partículas sólidas e manter o aglomerado adequadamente unido.

Um propelente compósito considerado típico tem uma formulação aproximada de $15 \%$ (massa/massa) de matriz polimérica, $65 \%$ (massa/massa) de cristais de oxidante (como o perclorato de amônio) e com uma distribuição modal das partículas variando entre 80 a 400 micrometros de diâmetro, e $20 \%$ de partículas de alumínio em pó, com distribuição de diâmetros entre 15 a 100 micrometros. A distribuição dos vários diâmetros de partículas, das espécies oxidantes e combustíveis, é projetada para maximizar a densidade do compósito assim obtido.

Durante um longo período de tempo acreditou-se que as formulações de propelentes sólidos tipo compósito fossem imunes ao potencial elétrico gerado por carregamento eletrostático. A ocorrência de acidentes com vários motores sólidos, sem causa aparente, obrigou a comunidade científica na área de propulsão química a estudar e ensaiar as diversas hipóteses para os acidentes e, com os resultados obtidos, concluiu-se que só restava a hipótese de ignição ou trinca por efeito do fenômeno de descarga eletrostática nesses motores. ${ }^{2}$ A eletricidade estática é conhecida há milhares de anos e é freqüentemente considerada como o efeito de cargas elétricas em repouso nos isolantes ou em condutores isolados, mas ainda não há um entendimento generalizado de como as cargas são transferidas de um material a outro quando um dos materiais é isolante. ${ }^{3}$ A concentração de partículas sólidas no compósito pode vir a aumentar a resistividade volumétrica do material se as partículas de alumínio estiverem recobertas por uma camada de alumina $\left(\mathrm{Al}_{2} \mathrm{O}_{3}\right)$, o que ocorre se a passivação do alumínio pelo óxido for completa. A camada de alumina torna a partícula de alumínio isolante e isto faz com que o valor de resistividade volumétrica do compósito seja maior do que a do polímero puro. ${ }^{4,5}$ A resistividade volumétrica (alta) define o propelente sólido compósito como um material dielétrico, isto é, um isolante que pode tornar-se condutor quando o seu campo de ruptura é ultrapassado pela aplicação de um campo elétrico exterior acima do limite de sua rigidez dielétrica (a máxima intensidade de campo elétrico que um dielétrico suporta sem ruptura é denominada de rigidez dielétrica e é uma propriedade intrínseca do material).

Esta característica dielétrica dos compósitos permite acumular cargas estáticas quando são submetidos a mecanismos de eletrização 
de materiais. Dentre os mecanismos mais estudados para propelentes sólidos compósitos temos: fracto-eletrificação, carregamento por contato (efeito triboelétrico) e radiação ionizante. Fracto-eletrificação e triboeletrificação são ações mecânicas, que ocorrem por efeito de atrito, fissuras no grão propelente sólido e descolamento entre o grão e o revestimento polimérico (liner) que serve de ligação com a parede do motor. A radiação ionizante induz cargas eletrostáticas no propelente, aumentando a voltagem armazenada no assim chamado grão propelente sólido. A probabilidade da ruptura elétrica sob radiação, de dielétricos de polímeros, é determinada pela condutividade elétrica no campo de ação da radiação, e é denominada Condutividade Induzida por Radiação - CIR. ${ }^{6}$

O potencial elétrico acumulado em uma determinada região do propelente compósito pode gerar uma descarga eletrostática onde o nível de energia é associado às condições ambientais, material e ao tempo de carregamento. ${ }^{7}$ A descarga eletrostática é freqüentemente associada à ocorrência de uma ruptura do dielétrico do ar atmosférico por uma superfície carregada, descarregando na terra ou em outra superfície de menor potencial.

Na ruptura, o campo elétrico libera elétrons acelerando-os e liberando outros elétrons por meio de colisões com átomos neutros ou moléculas. Rupturas de dielétrico ocorrem abruptamente (normalmente em um período de tempo de nano segundos) formando um caminho condutivo e uma descarga instantânea de alta energia através do material. Para polímeros não polares, a constante dielétrica é independente da freqüência da corrente alternada porque a polarização é efetivamente instantânea, polímeros não polares têm sempre constante dielétrica menor que três. ${ }^{8}$ No caso do propelente sólido compósito, a ruptura degrada severamente o dielétrico ou destrói sua capacidade isolante.

\section{GERAÇÃO DE CARGAS ELETROSTÁTICAS}

O mecanismo de geração de cargas elétricas em compósitos de emprego aeroespacial tem sido estudado mais recentemente com aplicações na área de materiais energéticos, como os propelentes sólidos compósitos, e em componentes eletrônicos. ${ }^{9}, 10$

Em um sistema compósito como o propelente sólido, ocorrem vários fenômenos físico-químicos. Dentre estes, o fenômeno associado com a troca de cargas livres através das interfaces metal/polímero/oxidante é o efeito que apresenta evidências de atuar como um formador de carga eletrostática. No compósito o metal normalmente agregado é o alumínio; o alumínio é um dos metais com o nível de Fermi próximo do nível energético do polímero. Na interface metal/polímero a troca de cargas livres torna possível equalizar as energias no nível de Fermi no metal e no polímero. Esta equalização faz ocorrer a formação de regiões de cargas livres em torno das partículas metálicas. ${ }^{11}$

Materiais compósitos de uma matriz polimérica com partículas de metal aleatoriamente dispersadas são considerados como um sistema heterogêneo desordenado. ${ }^{12} \mathrm{~A}$ formação de regiões de cargas livres em torno das partículas metálicas torna-se significante de acordo com a quantidade destas partículas na matriz polimérica.

Quando a densidade de partículas na matriz polimérica é suficientemente alta, as regiões de cargas no espaço, acompanhando diferentes partículas, se sobrepõem e o volume inteiro do bloco (metal/compósito) torna-se pobre ou rico de portadores carregados.

Alguns aditivos participantes da formulação do propelente sólido compósito podem capturar portadores de carga, mas o efeito mais significante é produzido pela presença das partículas de alumínio na formulação. As partículas sólidas, alumínio (Al) e perclorato de amônio (PA), alteram a característica de mobilidade das macromoléculas do polímero; o efeito primário é a restrição do número de possíveis conformações que as macromoléculas podem adquirir próximo à superfície. Esta restrição pode gerar estresse no compósito que por sua vez, em condições aleatórias, geram micro fissuras ou trincas no grão. ${ }^{9,13}$ Estas fissuras podem gerar cargas elétricas, que acumuladas devido às propriedades dielétricas do compósito, criam um potencial elétrico que se descarregado abruptamente tem energia suficiente para iniciar uma ignição com posterior processo de queima sustentada do propelente sólido. Da mesma forma, o acúmulo de cargas devido ao carregamento por contato, também pode gerar um potencial elétrico nas interfaces do material compósito.

Trabalhos desenvolvidos por Galembeck et al. ${ }^{14,15}$ demonstraram a possibilidade de moléculas de água serem polarizadas através de alta tensão $(>9 \mathrm{kV})$ transferindo carga a isolantes ou metais isolados, acoplado à evaporação da água, adsorção e dessorção. Metais isolados dentro de gaiolas de Faraday podem espontaneamente adquirir carga quando a umidade relativa está acima de $50 \%$, condição na qual o alumínio e o latão tornam-se negativos.

Os motores foguetes que empregam propelente sólido compósito utilizam o alumínio como combustível energético pelo aumento da variação da entalpia durante a queima do compósito. Este alumínio em pó é disperso na matriz polimérica e após a fabricação do grão propelente (um bloco cilíndrico com um vazio ao longo do eixo central) pode ocorrer a retenção de partículas de água no interior do compósito (o perclorato de amônio é higroscópico), mesmo sendo a resina de polibutadieno líquido hidroxilado $(\mathrm{PBLH})$ apolar, durante o armazenamento ou quando aguardando o uso operacional em ambientes com alta umidade. Esta retenção cria a possibilidade de geração de cargas relacionada às moléculas de água aprisionadas no compósito.

Os grãos propelentes são utilizados normalmente em condições adversas. Alguns exemplos: quando em posição de lançamento pode ocorrer atrito com partículas externas (vento e poeira) na estrutura do motor-foguete gerando cargas por fricção. Outro exemplo, a permanência em posição de lançamento (vertical) por um período longo pode gerar o descolamento de grão em relação à parede do motor, devido ao peso do mesmo, e induzir cargas por fractoeletrificação. Também pode ocorrer a indução de cargas elétricas através de radiação magnética ou térmica intencional ou natural. A voltagem resultante desta indução pode atingir um nível muito alto (acima de $20 \mathrm{kV}$ ) através da transferência de carga elétrica para as estruturas capacitivas parasitas no grão propelente.

Em estudos realizados com diversas formulações de propelentes sólidos compósitos de emprego aeroespacial, verificou-se que o comportamento elétrico do propelente é diretamente influenciado pelo percentual em massa de alumínio presente no compósito. ${ }^{5,16-18}$ Em simulações realizadas, ${ }^{4}$ observou-se que as partículas de maior diâmetro (400 micrometros) de perclorato de amônio (PA) forçam as partículas de alumínio a ficarem muito mais próximas do que o percentual previsto em projeto. Assim, um carregamento de alumínio de $20 \%$ (massa) numa formulação hipotética de propelente, a distribuição de volume entre as partículas de maior diâmetro de PA está aproximadamente localizada na fração teórica crítica do metal que é em torno de $29 \%$, e considerando que se as partículas de alumínio fossem perfeitamente condutivas, o valor de $29 \%$ de alumínio em um volume asseguraria que haveria um caminho de baixa resistência em qualquer amostra.

\section{Carregamento elétrico do compósito polimérico}

No estudo das características elétricas dos compósitos poliméricos os resultados experimentais, obtidos por diferentes pesquisadores, ${ }^{16-18}$ divergem parcialmente dos modelos teóricos do elemento de carregamento. Estudos recentes ${ }^{19}$ sugerem que a não consideração de vários fatores físico-químicos nos modelos, tais como a condutividade elétrica volumétrica específica do polímero puro, as energias 
de interação de polímero-polímero, polímero-partícula e partículas-partículas, concentração de partículas, tamanho médio e tipo de partículas, e as interações entre as macromoléculas, que influenciam o processo de formação de corrente elétrica nos compósitos poliméricos, são a principal razão desta divergência entre resultados experimentais e modelos teóricos.

Ao avaliar as condições de transferência de carga elétrica em polímeros condutivos, nas quais uma matriz polimérica com uma condutividade $\sigma$ p é preenchida com partículas com uma condutividade $\sigma$, verifica-se que o compósito resultante tem uma condutividade $\sigma$. A condutividade $\sigma$ está diretamente ligada ao fator de empacotamento $F$ das partículas. Quando a fração de volume das partículas atinge um valor crítico denominado de limiar de percolação, um aglomerado condutivo infinito é formado e o compósito torna-se condutivo. A percolação é uma teoria interdisciplinar da física e aplicada em diversos sistemas. Ela é o estudo da geometria dos agregados metálicos/meio/ isolantes e como as partículas afetam a ruptura do dielétrico quando aplicada em propelentes sólidos. Ye $e t a l^{20}$ afirmam que o fator de empacotamento $F$, Equação 1, é o limite de preenchimento do sistema, e é igual à maior fração de volume de partículas possível para um dado tipo de empacotamento dependente do formato da partícula.

Para um dado tipo de empacotamento, tem-se:

$$
F=V f /(V f+V p)
$$

onde $V f$ é o volume ocupado pelas partículas de preenchimento (Al e PA) e $V p$ o volume ocupado pela resina, ou matriz polimérica que aglomera essas partículas.

Para empacotamento de partículas esféricas de qualquer tamanho, estatisticamente monodispersas, o valor de $F$ é igual a 0,64 . O valor de $F$ diminui de acordo com o desvio no formato da partícula, e se forem consideradas as partículas polidispersadas o valor de $F$ pode crescer. Nos casos reais o valor de $F$ é bem menor que 0,64 ficando em torno de 0,59 a $0,62.5,21$

\section{PARTE EXPERIMENTAL}

Nas amostras de propelente consideradas nesse trabalho, estimou-se que as partículas têm a forma esférica conforme a representação esquemática na Figura 1. O propelente sólido utilizado nessa pesquisa teve como formulação básica a seguinte composição: aglomerante resina Polibutadieno Líquido Hidroxilado (PBLH), oxidante Perclorato de Amônia (PA) com perfil de distribuição granulométrico baseado nos diâmetros médios de 200 e 400 micrometros, aditivo acelerador de velocidade de queima $\left(\mathrm{Fe}_{2} \mathrm{O}_{3}\right)$ e combustível metálico alumínio (Al) com diâmetro médio de 18 micrometros. Os corpos de prova foram produzidos no formato cilíndrico com diâmetro de $10 \mathrm{~mm}$ e

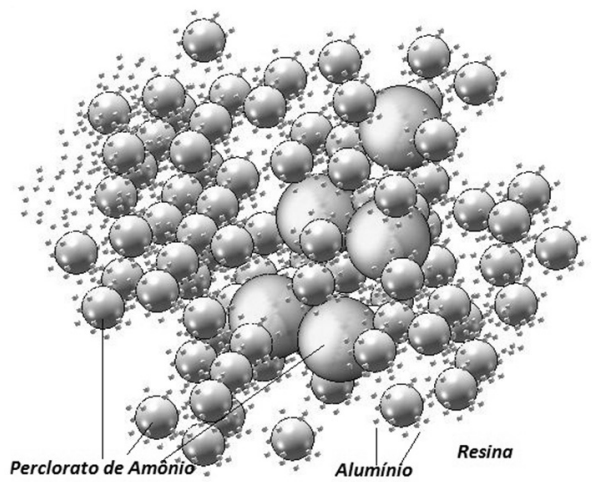

Figura 1. Representação esquemática do propelente sólido compósito e seus principais componentes químicos altura de $10 \mathrm{~mm}$. As superfícies das amostras, utilizadas para o contato elétrico com os eletrodos dos vários equipamentos de testes, foram preparadas utilizando-se uma camada de prata coloidal na superfície superior e inferior dos cilindros para obtenção de um contato elétrico uniforme em toda a superfície das amostras.

Os corpos de prova consistiram de cinco grupos de amostras de quatro formulações distintas, conforme apresentada na Tabela 1 a seguir.

Tabela 1. Formulação das amostras

\begin{tabular}{ccc}
\hline Grupo & Formulação & $(\%$ PA $)$ \\
\hline Prop A001 & PA/PBLH/Al - Bimodal & Carga $70 \%$ \\
Prop B001 & PA/PBLH/Al - Bimodal & Carga $75 \%$ \\
Prop C001 & PA/PBLH/Al - Bimodal & Carga $69 \%$ \\
Prop D001 & PA/PBLH/Al - Bimodal & Carga $\sim 69 \%$ \\
Prop E001 & PA/PBLH/Al - Trimodal & Carga 69\% \\
\hline
\end{tabular}

A Tabela 2 lista os equipamentos utilizados nas medidas das propriedades elétricas das amostras de propelente sólido compósito.

Tabela 2. Equipamentos dos ensaios

\begin{tabular}{cll}
\hline Item & Equipamento & Modelo \\
\hline 01 & High Resistance Meter & HP-4329 A \\
& & Hewlett-Packard \\
02 & LCR Meter & HP-3444 A \\
& & Hewlett-Packard \\
03 & Electrostatic Discharge Simulator & FTS-930D \\
& & Electro-tech Systems \\
04 & Microscópio Óptico LEICA & MZ95 Leica \\
& & Camera AG \\
05 & Microscópio Óptico Metalografico & DM5000M \\
& Leica & Leica Camera AG \\
06 & Microscópio Eletrônico de & LEO-15SS LEO \\
& Varredura - MEV & GmbH Oberkochen \\
\hline
\end{tabular}

Os ensaios foram realizados de acordo com o modelo CDM (Charged Device Model) especificado na norma MIL STD 1576 método $2205 .{ }^{22}$ Este modelo é aplicado conforme orientação da norma NASA MFSC-STD-1800-B ${ }^{23}$ e verifica a insensibilidade do propelente sob condições de uma descarga eletrostática. O procedimento prevê a aplicação de descarga eletrostática através de um capacitor de $500 \mathrm{pF}$ sem resistor em série (conforme Figura 2).

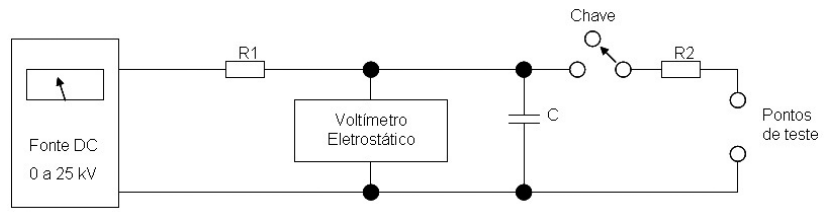

Figura 2. Diagrama em blocos do modelo CDM (Charged Device Model) para propelentes sólidos

O parâmetro de sucesso é que a amostra de propelente não pode ignitar ou deteriorar (trincas, fissuras) como resultado deste teste. O ensaio é realizado em condições de segurança, em área adequada por ser material ativo, usando o simulador de descarga eletrostática FTS-930D. Para os testes de armazenamento de carga eletrostática foi utilizado o mesmo modelo CDM. O teste é realizado injetando pulsos 
de $25.000 \mathrm{~V}$, com a capacitância intrínseca da amostra de propelente sendo utilizada como o capacitor do modelo, e com a resistência de descarga $\mathrm{R}_{2}$ de zero ohms (curto-circuito). É considerado o modelo mais severo dentre os modelos para teste de Descarga Eletrostática devido ao nível de energia utilizado para simular a descarga eletrostática. Os ensaios utilizaram 3 amostras de cada grupo, e a tensão residual foi monitorada depois de um período de 5 minutos após a série de injeção de energia através do Simulador de ESD (Electrostatic Discharge), para verificar o armazenamento de energia na amostra.

\section{RESULTADOS E DISCUSSÃO}

Nesse trabalho, o material estudado foi composto de amostras de diversas formulações (Tabela 1) de propelentes sólidos compósitos onde os principais componentes são: cristais de perclorato de amônio, alumínio em pó e polibutadieno líquido hidroxilado. Como fator adicional considerou-se que as partículas de alumínio sofrem oxidação e surge uma fina camada de alumina que possui propriedades elétricas isolantes. Esta camada é formada por auto passivação na partícula, com espessura na ordem de dezenas de angstroms. A alumina $\left(\mathrm{Al}_{2} \mathrm{O}_{3}, 99,9 \%\right)$ tem uma rigidez dielétrica em torno de $9 \mathrm{kV} /$ mm e esta rigidez é insuficiente para manter a característica isolante da partícula para os níveis de voltagem acumulados que ultrapassam facilmente estes valores. Aneli et al. ${ }^{19}$ consideraram a dependência do resistividade volumétrica do compósito em relação à quantidade de partículas condutivas, onde o aumento da quantidade de partículas de alumínio no compósito pode promover a junção de aglomerados isolados criando um canal de condutividade elétrica continuo, gerando uma região de transição de isolante para condutividade denominada transição de percolação estrutural isolante-condutor ou também conhecida como limiar de percolação.

Durante a análise das amostras com microscopia de varredura eletrônica foram identificadas aglomerações de partículas de alumínio dispostas aleatoriamente no material compósito conforme apresentado nas Figuras 3 e 4 . Estes aglomerados metálicos no compósito são formados devido a deficiências no processo de mistura (maceração), tais como a falta de passivação do alumínio antes do processo de mistura do compósito e a distribuição modal das partículas de PA com prevalência de grandes diâmetros (200, 400 mícron), o que contribui para o surgimento dos aglomerados devido aos vazios entre as partículas de PA durante o processamento. ${ }^{24,25}$ Estes aglomerados permitem o aparecimento da característica capacitiva que o propelente, aditivado com partículas metálicas, apresenta. Estes aglomerados com tamanho suficiente para serem considerados placas condutivas podem, através do contato entre várias aglomerações, criar altas capacitâncias

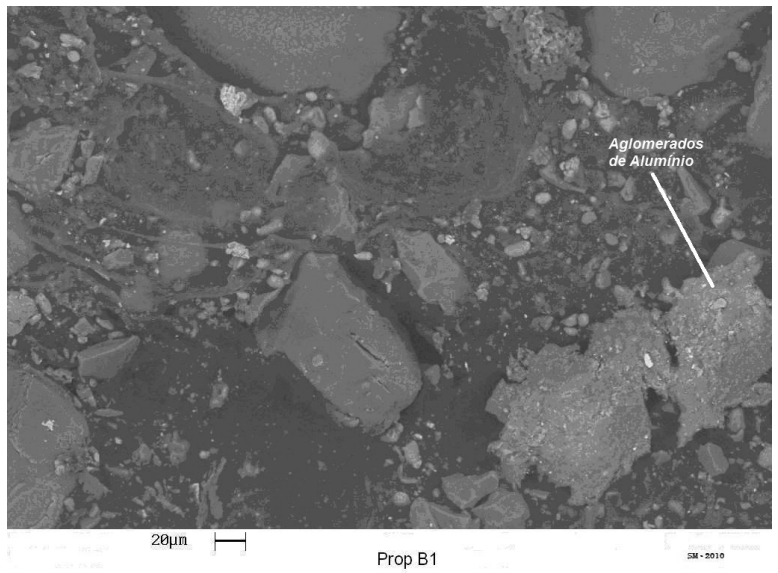

Figura 3. Aglomerados de alumínio presentes na composição do grão propelente sólido de uma amostra considerada nesse estudo - imagem MEV

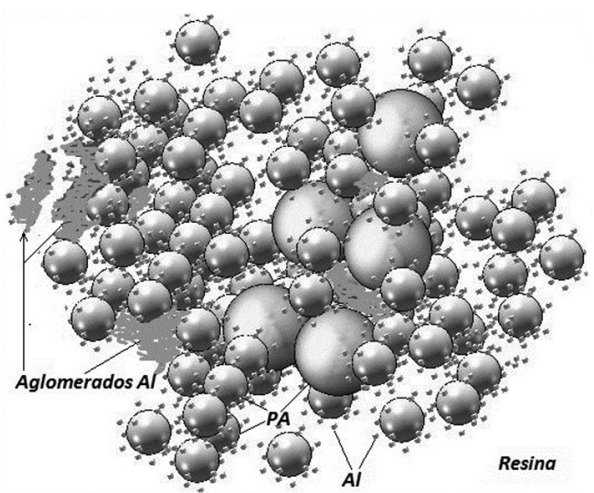

Figura 4. Representação esquemática dos aglomerados de partículas de alumínio no compósito

no propelente pelo aumento da capacidade de acúmulo de cargas elétricas, confirmando dados obtidos através da teoria de percolação para propelentes sólidos e de ensaios realizados. ${ }^{18,26}$

As amostras de propelente ao serem visualizadas no microscópio apresentam uma distribuição de partículas de cristais com formatos variados. Pode-se observar na Figura 5 (microscopia óptica) a distribuição modal das partículas de perclorato de amônio de uma determinada amostra de propelente. A imagem permite visualizar a quebra das partículas de PA que ocorre durante o processamento de obtenção do compósito, que é denominado maceração, gerando esta variedade de formatos.

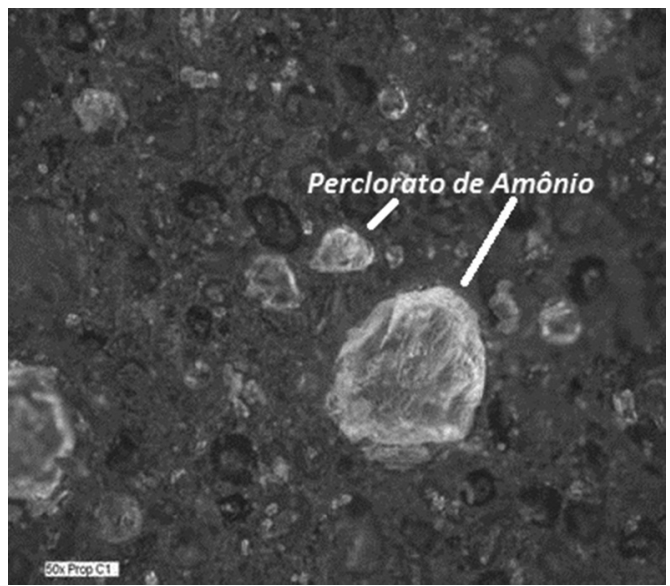

Figura 5. Imagem óptica da amostra do propelente COOI

A Tabela 3 apresenta os resultados de testes da injeção de energia nas amostras de propelente consideradas e a resistividade volumétrica das formulações.

Durante os ensaios na amostra B001, formou-se um arco elétrico entre a amostra e o aterramento, com a origem do arco no meio da

Tabela 3. Resultados de testes

\begin{tabular}{cccc}
\hline Amostra & $\begin{array}{c}\text { Quantidade } \\
\text { de Testes } \\
\text { V: } 25 \mathrm{kV} \\
\text { E: } 1,69 \mathrm{~J}\end{array}$ & Res. Vol. & $\begin{array}{c}\text { Voltagem } \\
\text { Residual } \\
\end{array}$ \\
\hline A001 & 30 & $3,9 \times 10^{11}$ & $>3 \mathrm{kV}$ \\
B001 & 30 & $4,2 \times 10^{10}$ & $>3 \mathrm{kV}$ \\
C001 & 30 & $7,4 \times 10^{11}$ & $>3 \mathrm{kV}$ \\
D001 & 30 & $9,1 \times 10^{11}$ & $>3 \mathrm{kV}$ \\
E001 & 30 & $6,7 \times 10^{11}$ & $>3 \mathrm{kV}$ \\
\hline
\end{tabular}


amostra. Os dados avaliados indicaram a presença de um aglomerado de alumínio na região. Este aglomerado desviou a tensão aplicada para a superfície lateral da amostra, ultrapassando a tensão de ruptura do ar (rigidez dielétrica) e ocasionando a abertura de um arco elétrico antes da base da amostra. Verificou-se, após inspeção microscópica óptica, a existência de um aglomerado metálico na região do arco. Este fenômeno indicou que a misturação do propelente não distribuiu de forma homogênea as partículas de alumínio na matriz polimérica conforme Figura 3.

A resina, juntamente com o oxidante, possui uma característica isolante muito alta, podendo considerar que no interior do grão propelente ocorre a formação de vários capacitores dispostos aleatoriamente. ${ }^{27}$ A provável ocorrência de capacitores próximos e paralelos pode armazenar uma quantidade de energia suficiente para ocasionar uma descarga eletrostática. As experiências realizadas injetando carga através de uma alta tensão $(25 \mathrm{kV})$ em amostras de propelente e medindo o decaimento da voltagem comprovaram a capacidade do propelente em reter carga por um período de tempo configurando a característica capacitiva do mesmo.

Devido à aleatoriedade da disposição das partículas no compósito, formato irregular, oxidação da superfície do alumínio, molhagem não homogênea das partículas (PA e Al) é extremamente complexo modelar o comportamento elétrico do grão propelente sólido. Beale et al. ${ }^{28}$ propôs um modelo de ruptura de dielétrico onde o processo ocorre via uma série de falhas microscópicas que leva a uma ruptura macroscópica e à uma descarga eletrostática. O campo elétrico necessário para causar esta completa ruptura é o mesmo campo que é necessário para causar uma falha microscópica.

A topologia considerada para o estudo da célula de armazenagem de cargas elétricas no propelente sólido compósito é a estrutura composta de dois aglomerados de partículas de alumínio separadas pelo polímero (agindo como dielétrico).

A topologia é considerada um capacitor padrão (metal/dielétrico/metal) e acontece quando ocorre a formação de aglomerados de alumínio com proximidades que caracterizem um capacitor, onde os aglomerados são as placas e o polímero é o dielétrico.

Os dados de resistividade volumétrica $(\rho)$ dos compósitos analisados apresentam um alto valor de resistividade. Esta resistividade alta faz com que a dissipação das cargas acumuladas ocorra de forma lenta, e sendo a velocidade de carregamento maior que a de descarga, o acúmulo de cargas pode atingir um potencial de voltagem muito alto. Durante os testes de injeção de cargas observou-se que pequena variação do percentual de alumínio (entre 15 e 20\%) não altera significativamente o tempo de permanência das cargas elétricas nas amostras. As resistividades das amostras apresentam valores compatíveis com os resultados das formulações usadas por Yeh et al..$^{29}$ onde os valores obtidos de resistividade intrínseca dos propelentes sólidos do tipo compósito em temperaturas entre $-50{ }^{\circ} \mathrm{C}$ $\mathrm{a}+70^{\circ} \mathrm{C}$, é apresentada com valores variando entre $5 \times 10^{13}$ a $2 \times 10^{9}$ $\Omega \mathrm{m} .{ }^{30}$ Covino et al. ${ }^{31}$ obtiveram valores entre $10^{13}$ a $10^{14} \Omega \mathrm{m}$ com amostras de propelente inerte (sem oxidante). Para propelentes ativos os valores foram de $5 \times 10^{10}$ a $129 \times 10^{10}$. Observaram também que a distribuição modal do PA causa a grande diferença entre os valores de resistividade medidos. A agência espacial norte-americana NASA (National Aeronautics and Space Administration), ${ }^{31}$ publicou no ano de 2008 recomendações para que os propelentes sólidos utilizados em seus propulsores tenham resistividade menor que $10^{9} \Omega \mathrm{m}$.

A maioria das amostras ensaiadas para este trabalho apresentaram uma variação entre $3,9 \times 10^{11}$ a $9,1 \times 10^{11} \Omega$ m e uma amostra apresentou um valor de resistividade de $4,2 \times 10^{10} \Omega \mathrm{m}$. O valor de resistividade é diretamente dependente do percentual de carregamento que é decorrente das dimensões das partículas sólidas, tempo de misturação e a fração de menor dimensão que surge resultante do atrito entre os cristais de PA durante o processo de formulação. Este percentual de carregamento e as variações modais das partículas sólidas podem explicar a diferença de magnitude entre o valores de resistividade dos compósitos PBLH/PA/Al encontrados na literatura $\left(10^{10}\right.$ a $10^{13}$ $\Omega \mathrm{m})$ e o valor obtido nas amostras $\left(10^{10} \mathrm{a} 10^{11} \Omega \mathrm{m}\right)$ utilizadas neste trabalho. Esta resistividade alta e as aglomerações de partículas de alumínio após o processamento do compósito são os principais fatores que explicam a capacidade do propelente sólido em armazenar cargas eletrostáticas.

\section{CONCLUSÕES}

Os dados de resistividade volumétrica $(\rho)$ apresentados neste trabalho, obtidos experimentalmente, nas várias composições das amostras demonstram que o compósito $\mathrm{PBLH} / \mathrm{PA} / \mathrm{Al}$ possui uma resistividade alta que é dependente da formulação e do tempo de mistura. Esse valor de resistividade alta contribui para que a dissipação das cargas armazenadas no compósito ocorra de forma lenta. Se a velocidade de dissipação é baixa, o acúmulo de cargas elétricas pode atingir um potencial muito alto. Este potencial acumulado, gerado pelas cargas elétricas, sendo descarregado de forma abrupta, pode gerar uma faísca elétrica com energia suficiente para ignitar de forma sustentada o motor foguete de um lançador de satélites ou de um armamento como um míssil.

\section{REFERÊNCIAS}

1. Sutton, G. P.; Biblarz, O.; Rocket Propulsion Elements, $7^{\text {th }}$ ed., Wiley: New York, 2001.

2. Manea, S.; Gonçalves, R. F. B.; Machado, F. B. C.; Iha, K.; Rocco, J. A. F. F; Iha, M. E. V. S.; AIAA Journal, 5258, p. 1-10, Denver, USA, 2009.

3. Williams, M. W.; American Scientist 2012, 100, 316.

4. Sunderaj, V. R. D.; Curry, J. D.; Larson, R. W.; National Symposium on Electromagnetic Compatibility - IEEE, Denver, USA, 1989.

5. Manea, S.; Tese de Mestrado, Instituto Tecnológico da Aeronáutica, Brasil, 2007.

6. Milekhin, Yu. M.; Sadovnichii, D. N.; Tyutnev, A. P.; Combustion, Explosion, and Shock Waves 2007, 43, 538.

7. Skinner, D.; Olson, D.; Block-Bolten, A.; Propellants, Explosives, Pyrotechnics $1997,23,34$

8. http://www.zeusinc.com/UserFiles/zeusinc/Documents/Zeus_Dielectric. pdf, acessada em Setembro 2007.

9. Deagan, R. D; Nagel, S. R.; Phys. Rev. B: Condens. Matter Mater. Phys. 1995, 52, 5653 .

10. Efimenko, K.; Rybka, V.; Svorcık, V.; Hnatowicz, V.; Appl. Phys. A 1999, 68, 479

11. Koropov, A. V.; Phys. Solid State 2004, 46, 1502.

12. Psarras, G. C.; Composites Part A 2006, 37, 1545.

13. Desai, S.; Thakore, I. M.; Sarawade, B. D.; Devi, S.; Eur. Polym. J. 2000, 36,711 .

14. Santos, L. P.; Ducati, T. R. D.; Balestrin, L. B. S.; Galembeck, F.; Proc. ESA Annual Meeting on Electrostatics, Cleveland, USA, 2011.

15. Ducati, T. R. D.; Simões, L. H.; Galembeck, F.; Langmuir 2010, 26, 13763.

16. Davenas, A.; Rat, R.; J. Propul. Power 2002, 18, 805.

17. Lee, R. J; Indian Head Division Naval Surface Warfare Center, IHTR 1925, Silver Spring, 1996.

18. Kent, R.; Rat, R.; Journal of Electrostatics 1985, 17, 299.

19. Aneli, J.; Zaikov, G.; Mukbaniani, O.; Chemistry \& Chemical Technology 2011, 5, 75 .

20. Mamunya, Ye. P.; Davydenko, V. V.; Pissis, P.; Lebedev, E. V.; Eur. Polym. J. 2002, 38, 1887.

21. McGeary, R. K.; J. Am. Ceram. Soc. 1961, 44, 513. 
22. MIL-STD-1576; Electroexplosive subsystem safety requirements and test methods for space systems, USAF, 1984.

23. MFSC-STD-1800-B; Electrostatic Discharge (ESD) Control for Propellant and Explosive Devices, NASA, 2008.

24. Anand, K. V.; Aviral, R.; Mulla, I.; Balbudhe, K.; Jayaraman, K.; Chakravarthy, S. R.; Proceedings of the Combustion Institute 2013, 34, 2139.

25. Tkaczyk, S. W.; Physica E 2009, 41, 1272.

26. Dilhan, K. M.; Birinci, E.; Yazici, R.; Karuv, B.; Walsh, S.; Polym. Eng. Sci. 2002, 42, 1609 .
27. Moon, K.; Pothukuchi, S.; Xu, J.; Wong, C. P.; Electronic Components and Technology Conference, Atlanta, Estados Unidos, 2002.

28. Beale, P. D.; Duxbury, P. M.; Phys. Rev. B: Condens. Matter Mater Phys. 1988, 37, 2785.

29. Yeh, W.; Budenstein, P. P.; IEEE Transactions on Dielectrics and Electrical Insulation 1995, 2, 84.

30. Wise, H.; J. Phys. Chem. 1967, 71, 9.

31. Covino, J; Hudson, F. E.; J. Propul. Power 1991, 7, 894 